${ }^{2}$ SMuTs, J. C. (1926) Holism and Evolution. New York: Macmillan. ${ }^{3}$ LIPOWsxi, Z. J. (1981) Holistic-medical foundations of American psychiatry. American Journal of Psychiatry, 138, 888-893.

${ }^{4}$ WITTKOWER, E. D. (1974) Historical perspective of contemporary psychomatic medicine. International Journal of Psychiatric Medicine, 5, 309-319.

${ }^{5}$ TUKE, G. H. (1872) Illustrations of the Influence of the Mind upon the Body in Health and Disease. London: Churchill.

6Alexander, F. (1950) Psychosomatic Medicine. New York: W. W. Norton.

'ShEPHERd, M. (1971) Book review of Modern Trends in Psychosomatic Medicine, 2, (ed. O. W. Hill) Journal of Neurological/ Neurosurgical Psychiatry, 34, 207.

SWWEeNEY, G. H. (1962) Pioneering general hospital psychiatry. Psychiatric Quarterly, (Suppl. part 2), 36, 209-268.
'LIPOWSKı, Z. J. (1974) Consultation-liaison psychiatry-an overview. American Journal of Psychiatry, 131, 623-630.

${ }^{10} \mathrm{HeNRY}$, G. W. (1929) Some modern aspects of psychiatry in general hospital practice. American Journal of Psychiatry, 86, 481-499.

${ }^{11}$ MAGUire, G. P. (1974) Psychiatric morbidity and referral in two general medical wards. British Medical Journal, 1, 268-270.

12 (1968) Physical illness in psychiatric patients. British Journal of Psychiatry, 113, 1365-1369.

${ }^{13}$ ShEPHERD, M. (1960) Psychiatric illness in the general hospital. Acta Psychiatrica et Neurologica Scandinavica, 35, 518-525.

${ }^{14}$ ANsTEE, B. H. (1972) The pattern of psychiatric referrals in a general hospital. British Journal of Psychiatry, 120, 631-634.

${ }^{15}$ LLoYd, G. G. (1980) Whence and whither 'liaison psychiatry'. Psychological Medicine, 10, 11-14.

\title{
Development of Mental Health Services
}

IAN B. Cookson, Consultant Psychiatrist, Sefton General Hospital, Liverpool and Chairman, Division of Psychiatry, Central/Southern Liverpool

In the Mersey Regional Health Authority it has been decided that closure of at least one large mental illness hospital will take place within some 10 years and may be complete by 1992 . To facilitate this the region has provided funding for every long-stay patient who might be discharged to the care of voluntary organisations or Social Services Departments and joint assessments of patients have been undertaken by the Health Service and Social Services staff.

Plans for the future of mental health services are advanced with regard to mental handicap and many subspecialties within psychiatry but the Steering Group created by the Chairman of the Regional Health Authority to speed the move to the practice of community mental health has sought further detail of the requirements for general adult services.

Liverpool District Health Authority, formerly two districts, now serves a population of some 400,000 and the requirements of the region and its principal city have been examined, several categories of patients being identified by consultant psychiatrists in a questionnaire as unsuitable for long-term treatment in district general hospitals. They are those patients with chronic schizophrenic disorders resistant to drug treatments, those with chronic brain syndrome (below the age of 65) and those with brain damage, those chronic patients with borderline mental handicap and psychosis, those requiring physical nursing for disorders such as Huntington's Chorea, those requiring medium security and those with personality disorders requiring treatment in therapeutic communities. There is also a large group of patients in crisis who would be better dealt with in a residential and day centre for evaluation and treatment of morbid distress of psychosocial origin.
Assuming the new chronic schizophrenic patients would require some 65 beds, the chronic brain syndrome and brain-damaged patients some 25 beds, physical nursing some 15 beds, medium security some 10 beds, and personality disorder, under the age of 22 years, some 15 beds and over the age of 22 years some 15 beds, it seems that approximately 145 beds are required for Liverpool in wards or hostel wards in the community, under the care of psychiatrists in order that adequate psychiatric nursing may be provided for these patients; direction and coordination of medical, psychological and social input will continue as at present. These figures are derived from the Regional survey, 200,000 of the Liverpool population having been loaded $25 \%$ for the factor of deprivation which has been assumed to be responsible for the increased demand for mental health services in the central area of the city.

It is quite clear that adequate provision for the nonacute patients cannot be provided adequately in the average district health authority and the practice of general adult psychiatry must function at a supra-district level in the future, if it is not to be splintered further to the detriment of individual patients and of the profession by the development of yet more parallel services. Perhaps consultants in general adult psychiatry should all have a half-time special interest with responsibility for two district health authorities.

This problem is being addressed in the Mersey Region and it is hoped that Liverpool, with a population of approximately twice that of an average district health authority, may act as a model for such developments. A central site, perhaps a complex of single-storey units for no more than 10 or 15 patients might be a new plan for the grouping of hostel wards in the community for patients not well 
enough for discharge from psychiatric inpatient care. It would facilitate nurse training, teaching of undergraduate medical students and other health professionals as well as providing a more homely atmosphere for patients than that of the district general hospital.

It appears that on closure of a large psychiatric hospital, one should provide for the most dependent patients first and create around them a mental health service uniting its various practitioners, other medical specialists, and the primary care service with other caring professionals and voluntary organisations. Clearly relationships with voluntary organisations and Social Services Departments need to be developed to ensure continuing access of patients of a kind previously resident in the large psychiatric hospital for long-term care. Facilities for those patients will now be funded from the rundown of the large hospital and supplementation from the Department of Health and Social Security. Access to such facilities must continue to enable the minimal non-acute provision and the acute provision, including that for the functionally ill elderly psychiatric patient of 0.44 per thousand, to operate efficiently.

A district mental health service to provide service requirements, to plan, budget and manage, to provide monitoring and facilitate training, teaching of undergraduates and postgraduates and research, would best function if the component of the health service, social services and voluntary organisations were operating as a single unit. This may have to be accomplished at an operational rather than an administrative level. Both Working Parties and Management Committees of services funded from the rundown of the psychiatric hospital and new joint ventures should have on them the Community Physician responsible for mental health liaison, Social Services mental health executive, Community Health Council secretary, representatives of voluntary organisations, a district health authority member, a city councillor and medical, nursing and other professional representatives of those operating the mental health service, particularly the Consultant Psychiatrist responsible for rehabilitation and the Psychiatric Nursing Officer responsible for residential and community psychiatric nursing services.

Research into evaluation of neuroleptic treatment in chronic schizophrenia, social deprivation in the inner city and consequences for mental health services, and evaluation of an eight-bedded unit for high dependency patients who might be discharged from the care of the psychiatrists, but continue on an outpatient basis with psychology, social work and psychiatric nursing in a residential setting, is clearly relevant to our planning in Liverpool at present.

The Royal College of Psychiatrists North-West Division had a meeting on Hospital Closure at Rainhill Hospital on 15 January 1986 and a further Rehabilitation Symposium took place on 7 March 1986 reviewing progress in the region, concentrating on the retraining and redeployment of psychiatric nursing staff.

No development as outlined above can be cheaper than the large psychiatric hospital and it is with some excitement we may look forward to beneficial change for our future patients and ourselves.

\section{ACKNOWLEDGEMENTS}

I am grateful to Dr David Enoch, Professor John Copeland, Dr Julian Leff and Dr John Howat for their time, interest and encouragement.

\section{Diploma in Geriatric Medicine}

In December 1985 the first examination was held for the Diploma in Geriatric Medicine. This examination is not intended for those aiming to be consultants in geriatric medicine but is intended for doctors wishing to contribute to the geriatric services of their district in a non-consultant capacity, in particular general practitioners wishing to do sessions as clinical assistants or hospital practitioners.

Psychiatric trainees who have spent a rotating placement in a psycho-geriatric unit might well wish to consider applying for the exam. A number of psycho-geriatricians are members of the Board of Examiners.

General practice trainees may find it convenient to take the Diploma during their vocational training (as for the
Diploma in Child Health). Entry qualifications include a period of experience in a hospital department providing specialist geriatric or psychogeriatric services.

The examination comprises both written and clinical sections and its emphasis is on the practicalities of patient management, particularly in community and primary care settings.

Doctors interested in taking the Diploma can obtain copies of the regulations, syllabus, specimen questions and suggested reading material from the Royal College of Physicians, 11 St Andrew's Place, Regent's Park, London NW1 4LE. 